\title{
IL FILOSOFO E LA GUERRA UN DIALOGO A DISTANZA FRA ERASMO E MORO
}

Gregorio Piaia*

SINTESE - Comparando os textos não muito longos de Erasmo e de Tomás Morus a respeito da guerra, percebe-se que este possui influências daquele. Para ambos, há uma clara e radical oposição entre a guerra e o filósofo. Para a guerra, são precisos corpos rudes, que não pensam; para a Filosofia, cérebros interrogadores. Morus parece encontrar mais facilidade em aceitar alguma forma de guerra, enquanto Erasmo vitupera mais acerbadamente a estupidez desta forma de violência.

\begin{abstract}
If we compare the short texts of Erasmus and Thomas More about the war, we see that the English statesman was influenced by the Dutch humanist. Both assert a clear and radical opposition between the war and the philosopher. War needs rough, not thinking bodies, while philosophy demands inquiring brains. However for More it seems easier to accept some manner of war, while Erasmus vituperates with more harshness the stupidity of such a violence.
\end{abstract}

Nella trama dell'affascinante iocus serius, intessuta di rimandi allusioni sottintesi ed ammiccamenti, con cui si costruisce il dialogo a distanza fra l'Encomium Moriae (nonché alcuni fra gli Adagia) e il "folle" racconto di Utopia, significativo e insieme problematico ci appare il tema della guerra: significativo per l'incisività della trattazione nei due autori e la sua persistente, tragica attualità; problematico perché al pacifismo moralistico di Erasmo sembra replicare, in Utopia, una visione della guerra ispirata alla più cruda Realpolitik, che dietro la maschera della razionalizzazione e umanizzazione lascia intravvedere - per citare il famoso libro di Gerhard Ritter - il "demoniaco del potere" (die Dämonie der Macht). ${ }^{1}$ Da qui il nostro

* Università di Padova.

1 Cfr. G. RITTER, II volto demoniaco del potere, tr. it., Bologna, Il Mulino, 1968 (II ed.), p. 88-94. Della vasta bibliografia sull'argomento ci limitiamo qui a citare le monografie di R. P. ADAMS, The Better Part of Valor. More, Erasmus, Colet, and Vives, on Humanism, War, and Peace, 1496-1535, Seattle, Univ. of Washington Press, 1962 e di PH. C. DUST. Three Renaissance Pacifists. Essays in the Theories of Erasmus, More and Vives, Bern-Frankfurt a. M.-New York, Peter Lang, 1988, oltre alle introduzioni apposte da due insigni studiosi dell'umanesimo alle loro sillogi di scritti erasmiani sulla pace: Guerre et paix dans la pensée d'Érasme, intr., choix de textes, commentaires et notes par J.C. Margolin, Paris, Aubier Montaigne, 1973, p. 7-25; E. GARIN, Erasmo, S. Domenico di Fiesole (Firenze), Edizioni Cultura della Pace, 1988, p. 5-54.

\begin{tabular}{|l|l|l|l|l|l|} 
VERITAS & Porto Alegre & v. 41 & $n^{0} 163$ & Setembro 1996 & p. 511-523 \\
\hline
\end{tabular}


intento di rileggere in sovraimpressione alcune celebri pagine erasmiane e moreane, facendo reagire il tema della guerra con quello del 'filosofo', meno appariscente ma non meno centrale: due temi che, accostati insieme al pari di due figure simboliche in un'incisione di Albrecht Dürer, sono in grado di offrirci parecchi elementi per chiarire il ruolo di Erasmo nella 'paternità' di Utopia.

A dire il vero, più che ai personaggi allegorici del Dürer è alle grottesche figure di Hieronymus Bosch che corre l'immaginazione, quando si rilegge il capitoletto che nell'Encomium Moriae è riservato alla follia della guerra: qui i soldati, "omaccioni grandi e grossi", muniti di grande audacia e di poco cervello, sono significativamente contrapposti agli esangui uomini di scienza, "qui studiis exhausti vix tenui frigidoque sanguine spiritum ducunt"; e non è certo a questi ultimi, "philosophis lucernariis" (adusi cioè a consumare gli occhi al lume di lucerna), che si può affidare "un'impresa così gloriosa" (res tam praeclara) quale la guerra, che è invece compito di "parassiti, ruffiani, briganti, sicari, contadini, imbecilli, indebitati e simile feccia umana". Ma Erasmo non si limita ad accomunare sarcasticamente soldati e sapienti sotto il comune stendardo della pazzia, al di là del diverso aspetto fisico. All'obiezione che anche in guerra v'è bisogno di cervello (consilium) egli risponde ch'esso è necessario solo in chi comanda, e in ogni caso si tratta di un consilium militare e non philosophicum: il distacco tra il filosofo e il soldato è per Erasmo incolmabile. $^{2}$

Si potrebbe pure obiettare che gli stessi filosofi combattono incessantemente tra loro (inexplicabilis inter ipsos est digladiatio), ${ }^{3}$ sia pure con le parole e non con le armi: un Bellum haudquaquam lachrymosum (come recita l'adagio 1523) , che vede scendere in lizza gli esponenti delle molteplici e rissose scuole filosofiche (albertisti, tomisti, scotisti, nominalisti...), ferocemente criticate da Erasmo. E nell'adagio Scarabeus aquilam querit non si rileva forse come a questo piccolo, disprezzato animaletto i Greci attribuissero una "singularis quaedam et incomparabilis sapientia", e gii Egizi vedessero in lui "un perfetto simbolo del guerriero ideale" (egregii bellatoris aptissimum symbolum)? Con sottile vena di umorismo (volta a sminuire la figura dell'aquila reale, che rappresenta lo sprezzante e sanguinario potere dei tiranni), Erasmo finisce cosi per conferire al povero scarabeo, solitamente associato allo sterco, le prerogative congiunte del sapiente e del condottiero... ${ }^{4}$

Rimane comunque ferma, per l'umanista olandese, la radicale incompatibilità fra l'intellettuale e il guerriero. Una incompatibilità che, espressa in tono canzonatorio nell'Encomium, si fa drammaticamente sofferta nell'adagio Spartam nactus est, hanc orna ("Ora che hai in mano Sparta, abbine cura"), la cui ultima parte è interamente dedicata alla rievocazione di Alessandro Stuart, figlio naturale del re di

2 Moriae encomium, id est Stultitiae laus, ed. C. H. Miller, Amsterdam-Oxford, North-Holland Publishing Co., 1979 (Erasmi Opera omnia, IV-3), p. 96, 464-477 (tr. it.: Elogio della pazzia, a cura di T. Fiore, intr. di D. Cantimori, Torino, Einaudi, 1972, cap. xxiii, p. 37-38.

3 Moriae encomium, p. 144, 369.(tr. it., cap. lii, p. 88).

4 ERASMO DA ROTTERDAM, Adagia. Sei saggi politici in forma di proverbi, a cura di S. Seidel Menchi, Torino, Einaudi, 1980, p. 164-165 e 168-173. 
Scozia e giovanissimo arcivescovo di St Andrew, "ad cognitionem omnium disciplinarum acerrimum" (era stato allievo di Erasmo in Italia), caduto col padre in battaglia contro gl'Inglesi nel 1513. "[...] ma che c'entri con Marte tu, adepto delle Muse, o meglio, adepto di Cristo? [...] Che ci fa l'uomo di scienza sul campo di battaglia (Denique quid erudito cum acie)? Che fa un vescovo con le armi in mano?", si chiede Erasmo con commossa deplorazione. ${ }^{5}$

Al di là di questi accenti personali, la condanna della guerra trova la sua formulazione più organica - prima ancora che nella Querela pacis (1517) - nel famoso adagio Dulce bellum inexpertis ("Chi ama la guerra, non l'ha vista in faccia"), ch'era stato ristampato con alcune aggiunte nell'edizione frobeniana degli Adagia, apparsa nello stesso anno (1515) in cui il soggiorno nelle Fiandre ispirò a Tommaso Moro la composizione di Utopia. Ma se il pacifismo dell'umanista olandese, come notava il Ritter, è contrassegnato da un "verboso pathos" e da un "moralismo estremistico", proprio di una "sensibile anima bella" che conosce assai poco il mondo del potere, il londinese Moro non appare certo "un sognatore pacifista del genere di Erasmo". ${ }^{6}$ Quella che ci è offerta nel libro II di Utopia sotto il titolo "De re militari" è in effetti una vera e propria 'filosofia della guerra', che a prima vista risulta assai difforme dall'indagine philosophicis oculis ${ }^{7}$ svolta da Erasmo sulla genesi della guerra e sui suoi perniciosi effetti, e più avvicinabile semmai a una certa tradizione 'italiana'. Più che il Machiavelli, il cui richiamo è sin troppo scontato, ci viene qui in mente un quesito che s'era posto il Boccaccio a proposito del canto IV dell'Inferno, ove tra gli "spiriti magni" trovano posto, accanto ai poeti e ai sapienti, i condottieri e le donne illustri: "Che hanno a fare - entro il "nobile castello" del Limbo - gli uomini d'arme con coloro li quali per filosofia son famosi?". Al che il Boccaccio risponde appellandosi a una concezione 'non professionale' della filosofia, intesa come la capacità di ragionare correttamente e in quanto tale presente a buon diritto sia nella strategia militare sia nel governo della casa. ${ }^{8}$ Inevitabile, a questo punto, il paragone con la philosophia civilior di Moro, contrapposta all' astratta philosophia scholastica...

Eppure, rileggendo il lunghissimo Dulce bellum inexpertis, si nota come alla fin fine Erasmo sconfessi, sia pure a denti stretti, quanto aveva sino ad allora sostenuto, adottando un procedimento retorico - una sorta di retractatio - analogo a quello messo in atto a conclusione dell'Encomium Moriae e della stessa Utopia (quando Moro accenna alla superbia quale ostacolo insuperabile alla diffusione dei felici ordinamenti di quell'isola beata): "Se poi la guerra è assolutamente inevitabile - perché grande è il numero dei malvagi - allora dopo aver tentato tutte le vie, dopo aver bussato a tutte le porte per amore della pace, la soluzione migliore sar

\footnotetext{
Ibi, p. 54-55.

RITTER, II volto demoniaco del potere, p. 59-61.

Adagia, p. 200.

8 Esposizioni sopra la Comedia di Dante, a cura di G. Padoan, in Tutte le opere di Giovanni Boccaccio, Milano, Mondadori, 1974, VI, p. 278-279.
} 
disporre acché l'iniqua faccenda sia gestita per mano d'iniqui e sia portata a termine con il minimo spreco possibile di sangue umano". ${ }^{9}$

Non è inverosimile che questo brano possa aver ispirato il capitolo "De re militari", ove Moro con tono serio ed insieme ironico ci presenta una societ in cui la guerra viene veramente condotta con il minimo dispendio di sangue, all'insegna di un rigoroso principio di economia: illusione (e follia) della guerra 'pulita' e a buon mercato... In effetti, se confrontiamo più da vicino il Dulce bellum inexpertis e le pagine di Utopia sulla guerra, si coglie un insistente giuoco di variazioni sul tema e di rovesciamenti di senso, che suonano come altrettante 'soluzioni' di quel bello spirito di Thomas More ai gravi problemi sollevati dall'amico Erasmo. Quest'ultimo, ad esempio, aveva sottolineato l'inconciliabilit della natura umana con la pratica della guerra: per la sua stessa "conformazione esteriore" - privo com'è, a differenza degli altri animali, di organi specifici atti alla difesa o all'attacco - è chiaro come l'uomo sia stato creato "non per la guerra ma per l'amore (non bello sed amicitiae), non per lo sterminio ma per la salvezza, non per fare il male ma per fare il bene". E non è tutto: "Non ancora contenta, la natura ha concesso all'uomo, esclusivamente all'uomo, la prerogativa della parola e della ragione (sermonis et rationis usum), che contribuisce moltissimo a stabilire ed alimentare l'amicizia (benevolentiam): e questo perché in nessuna circostanza l'uomo abbia bisogno di far ricorso alla violenza (ne quid omnino per vim inter homines gereretur)". E più avanti: "Ma se vuoi saper che cosa bestiale (ferina), vergognosa, disumana (non digna hominis) è la guerra, dimmi, hai mai assistito a uno scontro fra un leone e un orso? Fauci spalancate, ruggiti, brontolii sordi, ferocia, carneficina. [...] Ma quanto più vergognoso, quanto più crudele è lo spettacolo di due uomini che si fronteggiano, muniti di tante armi di offesa e di difesa! Chi li crederebbe uomini, dimmi, se l'abitudine al male (consuetudo malis) non ci avesse tolto la facoltà di meravigliarci?". ${ }^{10}$

La replica di Moro è chiara: in apertura del capitolo egli precisa anzitutto che gli Utopiani "Hanno in sommo orrore la guerra, cosa in tutto belluina (beluinam), ma che nessuna specie di belve pratica con tanta frequenza quanto l'uomo [...]". Ribaltando poi l'erasmiana hominis imaginem, che fondava il pacifismo sulla differenza radicale fra l'uomo e gli altri animali, egli pone proprio nelle capacità razionali (ingenii viribus), in quanto distintive dell'uomo, l'organo abilitato a fare la

9 Adagia, p. 280-283: "Quod si vitari nullo pacto potest ob multorum improbitatem, ubi nihil intentatum reliqueris, posteaquam pacis studio omnem moveris lapidem, tum proximum erit dare operam ut mala res per malos geratur et quam minimo dispendio humani sanguinis conficiatur". V. pure $\mathrm{p}$. 256: "Doctor vere christianus nunquam bellum probat; fortassis alicubi permittit, sed invitus ac dolens". Lo stesso principio viene ribadito nel capitolo "De bello suscipiendo" della Institutio principis christiani, apparsa a Basilea nel 1516 pochi mesi prima che Utopia venisse alla luce a Lovanio: "Bonus princeps nunquam omnino bellum suscipiet, nisi cum tentatis omnibus nulla ratione vitari potuit. Hoc animo si fuerimus, vix unquam existet inter ullos bellum. Denique si vitari non potest rem tam pestilens, tum proxima cura fuerit principis, ut quam minimo suorum malo, quam minimo Christiani sanguinis impendio geratur, et quam potest ocysissime finiatur" (Institutio principis christiani, ed. O. Herding, Amsterdam, North-Holland Publishing Co., 1974 [Erasmi Opera omnia, IV-1], p. 213-214, 463-467; tr. it.: L'educazione del principe cristiano, a cura di M. Isnardi Parente, Napoli, Morano, 1977, p. 156-157).

10 Adagia, p. 200-203 e 211-212. 
guerra e a condurre alla vittoria con i minori costi possibili. "Dicono infatti [gli Utopiani] che con la vigoria del corpo lottano gli orsi, i leoni, i cinghiali, i lupi, i cani e le altre bestie selvagge, le quali quasi tutte ci superano in fatto di robustezza e di aggressivit (robore ac ferocia), ma che ci sono tutte quante inferiori per intelligenza e raziocinio (ingenio, et ratione)". ${ }^{11}$

$\mathrm{E}$ ' questo approccio razionale (e insieme pragmatico) al 'fenomeno guerra' che rende attuabile l'accorata esortazione di Erasmo affinchè si sparga il minor sangue possibile. "Di una vittoria sanguinosa - dichiara infatti Moro con ironica serietà [gli Utopiani] non solo provano rammarico ma anche vergogna, perché ritengono da sciocchi aver acquistato a prezzo troppo alto una merce, per pregiata che sia; menano invece gran vanto se riescono a battere e sottomettere il nemico con stratagemmi ed inganni (arte doloque) [... $]^{1 .}{ }^{12} \mathrm{E}$ cosi, mentre Erasmo aveva deplorato l'introduzione della frode in guerra quale fattore di degenerazione, rimpiangendo l'epoca in cui si ricorreva soltanto alle armi comuni e al valore personale, ${ }^{13}$ Moro sembra divertirisi ad illustrare nei dettagli le tecniche raffinate e perverse (propaganda sovversiva, terrorismo, assassinio dei capi avversari grazie alla corruzione e al tradimento, appoggio alle fazioni interne, azioni di commando...) con cui gli Utopiani riescono a concludere le loro campagne militari "alla svelta (mature), sempre per badando piuttosto ad evitare i rischi che a guadagnarsi elogi o rinomanza". ${ }^{14}$

L'obiettivo, insomma, giustifica i mezzi... Ma è un obiettivo che riguarda pur sempre il "minimum dispendium humani sanguinis" auspicato da Erasmo come male minore, e che era già stato enunciato da Moro in un altro contesto, trattando dell'atteggiamento che gli Utopiani nutrono verso il denaro e i metalli preziosi. ${ }^{15}$ Moro ha tutta l'aria di voler esplicitare sino in fondo tale principio umanitario in sè accettabilissimo, mostrando paradossalmente com'esso conduca ad azioni inammissibili per la nostra morale, ma tenute in grande considerazione in quella 'società rovesciata' che è Utopia: un segno ulteriore, qualora ce ne fosse bisogno, dell'ambivalenza e "follia" del comportamento umano. Così, nel porre in risalto l'estrema generosità con cui gli Utopiani ricompensano i traditori in campo avverso, egli non esita a far presente che "Un tale sistema di mettere all'asta e di mercanteggiare la vita dei nemici (Hunc licitandi mercandique hostis morem) le qui vien da pensare, per ironico contrasto, al dialogo di Luciano I filosofi all'incanto...], da altri popoli esecrato come nefandezza crudele di menti degenerate, lo considerano molto onorevole, perché dà loro vanto non solo di avvedutezza, in quanto consente di liquidare quasi senza combattere le guerre più immani, ma anche di umanità e di miseri-

11 Utopia, ed. by E. Surtz and J. H. Hexter, New Haven and London, Yale Univ. Press, 1965 (The Complete Works of St. Thomas More, IV), p. 198, 30-31; 202, 20 -22 (tr. it. a cura di L. Firpo, Vicenza, Neri Pozza, 1978, p. 185 e 189). Utopia, p. 202, 12-15 (tr. Firpo, p. 189).

13 Adagia, p. 218: "Telis vulgaribus et virtute non dolo res agebatur".

14 Utopia, p. 202, 27-28 (tr. Firpo, p. 191).

15 Utopia, p. 148, 28 - 150, 9 (tr. Firpo, p. 129), ove si racconta che gli Utopiani, pur disprezzando l'oro, accumulano un tesoro inestimabile per far fronte ad eventuali guerre. "Satius est bellum pecunia aut arte declinare, quam multa sanguinis humani iactura gerere", si avverte, a mo' di sentenza, nel titolo marginale. 
cordia, perché con la morte di pochi colpevoli salvano la vita di numerosi innocenti (qui paucorum nece noxiorum, numerosas innocentium vitas redimant), che sarebbero stati destinati a cadere sul campo di battaglia, sia tra le proprie schiere che in quelle del nemico: una massa di popolo, quest'ultima, - insiste Moro con accenti decisamente erasmiani - che merita compassione non minore di quella dovuta alla propria gente, ben consapevoli come sono del fatto che sono entrati in guerra non già di propria iniziativa, ma trascinati dalla pazzia del re (principum furijs)" ${ }^{16}$

Erasmo aveva pure invitato ad affrontare quella cosa malvagia che è la guerra ricorrendo a individui malvagi (una sorta di applicazione sociale dei princip della medicina omeopatica...). Questi "iniqui" sono i soldati di mestiere, che poco prima aveva definito "barbari" e "infima feccia dell'umanità", dediti agl'incesti, agli omicidi, alle ruberie. ${ }^{17} \mathrm{E}$ Moro, puntualmente, si sofferma con sarcastico compiacimento sull'impiego dei mercenari - gli Zapoleti, ossia i "venditori della vita" - pagati profumatamente per scannare e farsi scannare (giacchè gli Utopiani, pur addestrandosi sempre nelle armi, scendono in campo aperto solo in casi estremi). Un impiego che non è poi molto dissimile da quanto avveniva nelle guerre del primo Cinquecento con i temuti e odiati mercenari svizzeri, ma che in Utopia raggiunge risultati veramente ottimali, giacchè molti di quei soldatacci comprati e mandati allo sbaraglio muoiono in battaglia, contribuendo cosi a liberare la terra "da quella caterva di scellerati sanguinari (tota illa colluvie populi iam tetri, ac nepharii)". ${ }^{18}$ Una sconcertante lezione di cinico machiavellismo da parte di Moro? Non crediamo proprio; anzi, il travestimento canzonatorio di un problema serio - e qui Moro si mostra più erasmiano di Erasmo - è il modo più efficace per imporre tale problema all'attenzione di un pubblico colto e smaliziato.

Nella sua idealizzazione del mondo antico, polemicamente contrapposto alle violenze sterili della moderna società cristiana, Erasmo aveva inoltre rilevato che i principi pagani erano soliti civilizzare i territori conquistati e fondare città nelle zone disabitate (regiones incultas extructis oppidis reddebant celebres), migliorando le condizioni di vita degli abitanti, sicchè a quei tempi era conveniente perdere la guerra (ut tum expedierit devinci)" ${ }^{19}$ Moro, dal canto suo, accenna alla politica di colonizzazione messa in atto dagli Utopiani, quando il sovrapopolamento della loro isola li induce a trasferire parte degli abitanti nel vicino continente, "dovunque ci siano distese di terre non utilizzate (ubicumque indigenis agri multum superest)". Qui si fondono con gli indigeni, ai quali trasmettono le loro istituzioni, e "con i loro metodi riescono a far s che una terra, che in passato si mostrava avara ed ostile a un solo popolo, adesso risulti più che sufficiente per due". La situazione non è però sempre idilliaca, poichè gl'indigeni che rifiutano di assimilarsi sono cacciati dalle loro antiche terre; e poi, "Contro chi fa resistenza muovono guerra, poichè considerano che non ci sia causa bellica più giusta di quella d'un qualsiasi popolo, che si trovi in possesso di un territorio, che esso non mette a frutto, mantenendolo inerte

16 Utopia, p. 204, 18-26 (tr. Firpo, p. 191-193).

17 Adagia, p. 279. Sui mercenari, "barbarica fex sceleratissimorum hominum", v. pure l'Institutio principis chnstiani, p. 214, $478-481$ (tr. it., p. 157).

18 Utopia, p. 208, 10-14 (tr. Firpo, p. 195).

19 Adagia, p. 250-251. 
e deserto, e ne impedisca l'utilizzazione ad altri che per legge di natura (ex naturae prescripto) hanno bisogno di trarne il proprio nutrimento"..$^{20}$

Il tema della guerra di espansione per motivi economici era già presente in Platone (Resp. II, 373d-e), ma non è da escludere che pure in questo caso Moro intendesse 'rispondere' ad Erasmo, muovendo da uno spunto paradossale proposto da quest'ultimo (nell'antichità a certi popoli conveniva perdere la guerra...) per poi ribaltare la prospettiva di fondo (il pacifismo erasmiano) sino a conferire piena legittimità alla guerra coloniale. Se il letterato e teologo Erasmo contesta il principio della "guerra giusta", riconosciuto dal diritto romano, ${ }^{21}$ il giurista Moro non sembra infatti aver difficoltà a considerare pienamente lecita la guerra quando sia motivata da ragioni plausibili (lo sfogo alla sovrapopolazione, la difesa del proprio paese e dei paesi alleati o amici, la punizione degli assassini di cittadini utopiesi...). ${ }^{22} \mathrm{Ma}$, a ben vedere, il divario fra i due umanisti si riduce se si tien presente che in Utopia sono venute meno le ragioni che, per Erasmo, si trovano all'origine della maggior parte delle guerre che imperversano nella cristianità: "nos aut puerilis ira aut pecuniae fames aut gloriae sitis, saepenumero foeda merces huc adducit", deplora l'umanista olandese, ponendo a confronto i cristiani del suo tempo con l'antico Israele, che combatteva, sì, ma contro i popoli idolatri suoi nemici, ${ }^{23}$ e poco più avanti, usando l'immagine della guerra in senso spirituale, egli rammenta che "da quando Cristo dette l'ordine di riporre la spada [cfr. Matth. 26, 52; Joh. 18, 11], al cristiano non è lecito combattere che la più gloriosa delle guerre, diretta contro i più spietati nemici della chiesa: la cupidigia, l'ira, l'ambizione, la paura della morte (pulcherrimum illud praelium cum teterrimis hostibus ecclesiae, cum studio pecuniae, cum iracundia, cum ambitione, cum metu mortis). Ecco i nostri Filistei, ecco i Nabuccodonosor, Moabiti ed Ammoniti ai quali non bisogna dar tregua [...]: solo questa guerra genera la pace vera (Solum hoc bellum veram gignit pacem). Chi ne esce vincitore, non vorrà più saperne di combattere con esseri umani". ${ }^{24}$

Ed ecco la risposta paradossale che si deduce dall'"aureo libello" di Tommaso Moro: in Utopia le condizioni per l'instaurarsi di una vera pace si sono realizzate ancor prima che i suoi abitanti venissero a contatto del messaggio cristiano. In effetti il regime e lo stile di vita degli Utopiani fanno s che i loro governanti siano del tutto alieni dai fanciulleschi scoppi d'ira e dall'inconsulto desiderio di gloria, che così spesso inducono i sovrani d'Europa a guerre disastrose; l'assenza di proprietà privata e di circolazione monetaria - riprenderemo più avanti questo tema - rende impossibile la funesta brama di denaro, mentre la pratica mercenaria (foeda merces) è interamente piegata, come s'è visto, alle esigenze di razionalizzazione della guerra, inserendosi in un meccanismo perfetto e implacabile.

20 Utopia, p. 136, 13-17 (tr. Firpo, p. 115).

21 Adagia, p. 242-243; v. inoltre p. 258-261, ove si rifiuta la guerra punitiva, anche se dettata da motivi di giustizia, perchè dà luogo a ingiustizie ancor maggiori: "Ouod si quis clamet iniquum non dare poenas qui peccarit, respondeo multo esse iniquius tot innocentum millia citra meritum in extremam vocari calamitatem".

Utopia, p. 200, 25 - 202, 12 (tr. Firpo, p. 187 e 189).

Adagia, p. 250-251.

Ib., p. 252-253. 
Ma perfino il timore della morte, se non proprio vinto, è di gran lunga ridotto negli Utopiani, che accanto all'addestramento militare ricevono un'accurata preparazione psicologica e 'filosofica', che li porta a "non [...] disprezzare la vita al punto da arrischiarla sconsideratamente", ma neppure ad amarla "senza misura, così da volerla preservare in modo sordido e meschino (avare turpiterque) quando l'onore suggerisce di metterla a repentaglio" ${ }^{25} \mathrm{E}$ non è soltanto la paura di morire in guerra che viene allontanata, ma anche la paura della morte tout court. Fiduciosi in massima parte nella felicità ultraterrena, gli Utopiani non si rattristano di fronte alla morte ed anzi gioiscono per coloro che muoiono "con lo spirito sollevato e colmo di speranza (alacriter ac pleni bona spe)", e questo "laetus transitus" viene posto al centro di ogni rievocazione del defunto. ${ }^{26}$ Ancora una volta gli abitanti dell'isolache-non-c'è manifestano un comportamento più genuinamente cristiano dei cristiani veri, anche se 'contaminato' con lo stile di vita - e soprattutto di morte - degli antichi saggi pagani, incarnato in particolare da Seneca e cosi spesso ricordato da Diogene Laerzio nella sue Vitae philosophorum (ed è in questa prospettiva che va collocato il tema, in sè sconcertante, della "mors spontanea" o eutanasia attiva). ${ }^{27} \grave{E}$ il caso di ricordare che nell'Encomium Moriae sono i matti e i deficienti ad essere considerati i più felici fra gli uomini, e proprio perché, anzitutto, non hanno paura della morte ("Principio vacant mortis metu")? ${ }^{28}$

$\grave{E}$ con questo spunto di riflessione che chiudiamo l'analisi della risposta 'filosofica' fornita in Utopia al tema della guerra, qual era stato trattato in particolare nel Dulce bellum inexpertis. Ma questo tema può ricevere luce ulteriore da un approfondimento del concetto di 'filosofo' (e quindi di 'filosofia') in Erasmo e Moro. In un altro celebre adagio, Sileni Alcibiadis, il modello del filosofo per eccellenza è visto in Socrate, brutto e ridicolo all'aspetto, ma nel suo intimo "essere più divino che umano", quali erano per l'appunto presso i Greci certe statuine intagliate che all'esterno presentavano figure sconce e grottesche, e all'interno immagini divine. Da filosofo vero, Socrate era indifferente alle ricchezze (frutto di strapazzi e di guerre), alle offese, alla fortuna, alla stessa paura della morte... 'Filosofo', quindi, non è chi porta il mantello e la bisaccia e si fa crescere la barba, ma chi va contro corrente, chi pensa differenziandosi dalle convinzioni più diffuse, ma non per questo più valide: cosi Erasmo proclama con forza, dopo aver citato il "divino" Platone, in un altro adagio 'politico', che vede beffardamente uniti insieme i re e i matti, Aut

Utopia, p. 210, $19-22$ (tr. Firpo, p. 199).

Utopia, p. 222, $30-224,2$ (tr. Firpo, p. 213).

Cfr. Utopia, p. 186, 3-19 (tr. Firpo, p. 171).

Moriae encomium, p. 114, 800 (tr. Fiore, cap. xxxv, p. 57).

Adagia, p. 62 e 64: "Atqui si Silenum hunc tam ridiculum explicuisses, videlicet numen invenisses potius quam hominem, animum ingentem, sublimem ac vere philosophicum, omnium rerum pro quibus caeteri mortales currunt navigant sudant litigant belligerantur, contemptorem, iniuriis omnibus superiorem et in quem nullum omnino ius haberet fortuna et usque adeo nihil timentem, ut mortem quoque nulli non formidatam contempserit, in tantum ut cicutam eodem ebiberit vultu quo vinum solet [...]". V. invece, a p. 78-79, il ritratto del falso filosofo, fanfarone ghiottone ed imbroglione. 
regem aut fatuum nasci oportere ("Re o matti si nasce"). ${ }^{30}$ Ed è significativo che questo stesso concetto compaia, oltre che nei Sileni di Alcibiade, ${ }^{31}$ nella dedica della Institutio principis christiani al giovane Carlo di Borgogna (il futuro Carlo V), con riferimento - nuovamente - alla sentenza di Platone, e in contrapposizione alla filosofia scolastica; insomma, una versione erasmiana del concetto di philosophia civilior, quale veniva contemporaneamente abbozzato in Utopia:

Proinde Plato non alia in re diligentior est quam in instituendis reipublicae suae custodibus, quos non opibus non gemmis non cultu non imaginibus non satellitio, sed sola sapientia caeteros vult antecellere negans unquam beatas fore respublicas, nisi aut philosophi gubernaculis admoveantur aut ii, quibus forte contigit administratio, philosophiam amplectantur; philosophiam, inquam, non istam, quae de principiis de prima materia de motu aut infinito disputat, sed quae falsis vulgi opinionibus ac viciosis affectibus animum liberans ad aeterni numinis exemplar recte gubernandi rationem commonstrat. ${ }^{32}$

Ė grazie agli "occhi filosofici" che Erasmo può cogliere e denunciare l'assurdità per cui la guerra, ossia la cosa più rovinosa, squallida e indegna dell'uomo, è ormai considerata "un fenomeno cosi largamente recepito, che chi la mette in discussione passa per stravagante e suscita la meraviglia" ${ }^{\prime 33}$ anzi, persino di fronte alle vergognose guerre fra cristiani "nessuno fa domande, nessuno si leva a dire una parola di riprovazione (Et o caecitatem mentis humanae, haec nemo miratur, nemo detestaturl)", e monaci e teologi e giuristi e perfino vescovi si mobilitano per giustificare o fomentare presso i príncipi queste lotte fratricide. ${ }^{34} \mathrm{E}$ Moro a sua volta (cui Erasmo nella dedica dell'Encomium Moriae aveva attribuito la dote del vero filosofo), ${ }^{35}$ nel riportare un'immaginaria seduta del consiglio del re di Francia, demistifica con pe-

30 Ibi, p. 10: "Neque enim temere divinus ille Plato scripsit non aliter felices fore respublicas, nisi si aut philosophis committeretur imperium, aut quibus imperium contigisset philosophiam sequerentur. Porro philosophiam sequi non est pallium aut peram circumferre, barbam demittere. Quid igitur? Ista quae vulgus stulte miratur contemnere deque rebus longe secus sentire, quam hominum multitudo sentit".

31 Ibi, p. 92: "[...] illud ostendo, vulgus hominum multo pluris ea facere, quae cemuntur oculis, quam quae hoc verius sunt, quo minus conspiciuntur"; v. pure p. 82-85, ove Erasmo fomisce un campionario delle "opinioni a rovescio proprie della multitudine", con la conseguente inversione dei termini e dei valori ad essi sottesi ("Deinde ex praeposteris opinionibus praepostera rerum vocabula. Quod excelsum est humile vocant, quod amarum dulce, quod preciosum vile, quod vita mortem"). Institutio principis christiani, p. 133-134, 16-24.

Dulce bellum inexpertis, in Adagia, p. 198-201: "Ouod si quicquam est in rebus mortalium quod constanter aggredi conveniat, imo quod oporteat modis omnibus fugere, deprecari, propellere, certe bellum est, quo non alia res vel magis impia vel calamitosior vel latius perniciosa vel haerens tenacius vel tetrior et in totum homine indigna, ut ne dicam christiano. [...]

Quibus rebus fit ut nunc bellum adeo recepta res sit ut demirentur homines esse cui non placeat [...]. Id quod magis etiam admirabitur quisquis animum a vulgo receptis opinionibus ad ipsam rerum vim ac naturam perspiciendam converterit ac seorsum hinc hominis imaginem, hinc belli simulacrum vere philosophicis oculis aliquantisper contempletur".

Ibi, p. 220-221 e 276-277.

Moriae encomium, p. 67-68, 16-19: "Quanquam tu quidem, ut pro singulari quadam ingenii tui perspicacitate, longe lateque a vulgo dissentire soles, ita pro incredibili morum suavitate facilitateque cum omnibus omnium horarum hominum agere, et potes et gaudes" (tr. Fiore, p. 4). 
netrante ironia le guerre del suo tempo e mostra com'esse siano frutto di ambizione ed avidit; un buon sovrano dovrebbe invece dedicare tutte le sue energie a migliorare le condizioni del regno ricevuto in eredità dai propri avi, piuttosto che sperperare risorse in una politica espansionistica o sbandierare la minaccia di guerra per spremere ulteriori tasse ai suoi sudditi. ${ }^{36}$

Ė un tema già presente nell'adagio Aut regem aut fatuum nasci oportere, ${ }^{37} \mathrm{e}$ soprattutto nell'altro adagio Spartam nactus es, hanc orna, che riguarda espressamente il dovere che ogni soviano ha di far prosperare il proprio regno - fosse pure piccolo e modesto - anzichè far guerra per impossessarsi dei regni altrui. "Nihil principi pulchrius quam ut hoc quicquid est regni, quod fortuna dedit, sua sapientia, virtute, diligentia reddat ornatius", aveva dichiarato Erasmo, riprendendo il verbo ornare del titolo; e Moro, di rimando: "[...] proinde avitum regnum coleret, ornaret quantum posset, et faceret quam florentissimum" ${ }^{38}$ Questo saggio principio viene illustrato da Erasmo con numerosi esempi tratti dalle vicende contemporanee, mentre Moro porta l'esempio del popolo degli "Acori" (ovvero dei "Senzaterra", con ironica allusione al regno di Francia) che dopo aver conquistato con le armi un altro regno si risolsero alfine ad abbandonarlo, tanto elevati erano i costi militari ed economici. Che poi Moro avesse ben presente questo adagio nella stesura del libro I di Utopia ci è confermato da una corrispondenza assai significativa, che sembra essere sfuggita agli editori critici. Ci riferiamo alla bella similitudine teatrale con cui egli illustra la philosophia civilior, che - a differenza della rigida ed ingessata philosophia scholastica - "conosce il suo palcoscenico e vi si sa adeguare, sostenendo con garbo e con decoro la sua parte nel dramma che si recita (quae suam novit scenam, eique sese accomodans, in ea fabula quae in manibus est, suas partes concinne et cum decoro tutatur)". Ma già Erasmo, in una giunta inserita nell'edizione 1515 degli Adagia, aveva notato:

Proverbium [i.e. Spartam nactus es, hanc orna] igitur ad varios usus licebit accomodare, vel cum admonebimus ut suam quisque personam, quam suscepit, cum decoro tueatur. ${ }^{39}$

Anche la successiva similitudine di Moro fra lo Stato e la nave in preda ai venti si pu accostare al paragone di Erasmo fra l'uomo saggio e il bravo timoniere; ${ }^{.0}$ così come l'invito di Moro al filosofo affinchè, se non è possibile guarire (mederi) i vizi della società, s'impegni a "ridurre almeno al minimo il male (efficias saltem, ut sit quam minime malum)" non è soltanto una replica alla secca dichiarazione di Itlodeo "non esse apud principes locum philosophiae" (che sembra riecheggiare le pa-

36 Utopia, p. 56, 21-26; 86, 22 - 90, 22 (tr. Firpo, p. 27 e 61-65).

37 Adagia, p. 18-21.

38 Ibi, p. 44; Utopia, p. 90, 17-18.

39 "Di questo proverbio si potrà far uso in varie circostanze, per esempio per ricordare agli altri che ognuno deve recitare dignitosamente la parte che si è assunto" (Adagia, p. 40-41); cfr. Utopia, p. 98, 12-14 (tr. Firpo, p. 73), e p. 372, ove in nota si fa riferimento ad altri Adagia e alla Moria.

40

Cfr. Utopia, p. 98, 27-28: "[...] non ideo tamen deserenda Respublica est, et in tempestate navis destituenda est, quoniam ventos inhibere non possis [...]"; Adagia, p. 42: "Quemadmodum enim peritus gubernator in omni tempestate bonum praestat nauclerum, ita vir sapiens in omni fortuna sapienter se gerit". 
role della Follia "Sed abhorrent a vero principum aures"). ${ }^{41}$ Tale invito si può leggere anche come una risposta 'operativa' all'esortazione che Erasmo - alla fine di questo adagio - rivolge al principe perché ponga rimedio, con il minor versamento possibile di sangue, alla lunga lista dei mali che affliggono la comunità. ${ }^{42}$ Per Moro solo con l'aiuto del filosofo, libero dai pregiudizi e dallo spirito di adulazione, il sovrano può puntare a quest'obiettivo; ciò richiede però il superamento della diffidenza che l'amico Erasmo nutre in genere versi i pr ncipi e le loro corti...

È questo, com'è noto, il tema dibattuto nel "dialogo sul consiglio", aggiunto da Moro alla primitiva stesura di Utopia e in cui la figura del filosofo appare problematicamente sdoppiata fra i due interlocutori, Itlodeo (che si fa portavoce della posizione di Erasmo) e lo stesso Moro. ${ }^{43} \mathrm{Ma}$ nel resto dell'opera è Itlodeo che incarna il filosofo vero, fornito di una vista più penetrante e latore di un racconto-messaggio che sovverte le opinioni correnti sull'origine dei mali sociali e quindi delle stesse guerre, per lo meno di quelle inutili e ingiuste. Tale origine risiede nel denaro e nella propriet privata, fonte d'ingiustizia, di miseria, d'infelicità, nonchè di quella vera e propria guerra civile che à rappresentata dalla quotidiana catena di furti rapine omicidi, invano combattuti con le esecuzioni capitali. ${ }^{44}$

Ebbene, i presupposti 'filosofici' di questa tesi "folle" e provocatoria si possono rintracciare - e qui torniamo al Dulce bellum inexpertis - proprio nell'indagine di Erasmo sulla guerra, in cui risulta evidente il nesso che unisce la brama di possedere e la pratica della guerra: Cristo predicò la pace e il disprezzo dei beni materiali; noi invece distorciamo e adattiamo le sue parole, quasi temessimo che una volta o l'altra possa scoppiare la pace e si smetta di rincorrere la ricchezza, o quasi volessimo tornare alle "leggi" che reggevano la società prima che fosse annunziato il Vangelo (punire, combattere, accumulare beni materiali). ${ }^{45}$ Gli Utopiani invece, che sono 'naturalmente' cristiani, hanno già messo in pratica l'invito a rinunciare alle ricchezze, tant'è vero che la loro adesione al cristianesimo predicato da Itlodeo e dai suoi compagni fu favorita dall'"aver essi appreso che Cristo approvò la vita in comune dei suoi e che questa viene tuttora praticata nelle più genuine convivenze cristiane". ${ }^{46}$

41 Cfr. Utopia, p. 98, 8-9; 100, 1-2; Moriae encomium, p. 116, 1.

42 Adagia, p. 56: "His ita mederi, ut in remedium quam minimum de sanguine tuorum impendas, ut respublica beneficium sentiat, non sentiat impendium, an non egregium principis munus et immortali laude dignum?".

43 Cfr. J. H. HEXTER, L'utopia di Moro. Biografia di un'idea, a cura di M. P. Fimiani, Napoli, Guida, 1975, p. 98-149.

44 Utopia, p. 242, 2-5: "Quis enim nescit fraudes, furta, rapinas, rixas, tumultus, iurgia, seditiones, caedes, proditiones, veneficia, cotidianis vindicata potius quam refrenata suppliciis, interempta pecunia commori [...]" (tr. Firpo, p. 233).

45 Adagia, p. 234-236: "Et quoniam intelligebat pacem aliter non posse constare, nisi penitus contemptis his pro quibus mundus hic digladiatur, iussit ut ab ipso disceremus esse mites. Beatos vocavit qui divitias [...] pro nihilo ducerent, nam hos vocat pauperes spinitu"; p. 254: "Et perinde quasi periculum sit ne quando mundus conquiescat a bellis, ex Christi verbis bellum asserimus; et perinde quasi metuamus ne relanguescat in congerendis opibus mortalium avaritia, Christum facimus auctorem congerendae rei, huc detorquentes illius verba quasi praeceperit, non permiserit, ea quae prius interdixerat. Mundus habebat suas leges antequam exoriretur Evangelium: puniebat, bellabat, congerebat in aerarium ac penum".

Utopia, p. 218, 5-8 (tr. Firpo, p. 207). 
È la philosophia Christi che traspare dietro il richiamo di Erasmo al discorso delle beatitudini e di Moro alla vita comunitaria della primitiva Chiesa di Gerusalemme (Atti, 2, 44-45; 4, 32-35) e degli ordini monastici fedeli alla regola. In linea con questa ispirazione, in precedenza Erasmo ha impresso al suo discorso - volto a 'spiegare' la graduale degenerazione dell'uomo - una decisa valenza antiaristotelica. Il disordine, la guerra e la corruzione sono penetrati nel popolo cristiano grazie alla cultura pagana (eruditio), inizialmente adottata per combattere gli eretici, e poi grazie ad Aristotele, accolto in teologia al punto da prevalere sulla stessa parola di Cristo, e al diritto imperiale romano, che legittima - come s'è detto - la guerra. In particolare, "Aristotele ci ha insegnato che la felicità dell'uomo non è perfetta, se non è corredata da un bell'aspetto e da beni di fortuna. Aristotele ci ha insegnato che non pu prosperare lo stato nel quale tutti i beni sono comuni (non posse florere rempublicam in qua sint omnia communia) (è la critica alle tesi platoniche, espressa da Aristotele in Polit., 1262b - 1263a]. E noi vogliamo accozzare il suo sistema con l'insegnamento di Cristo (Huius omnia decreta cum Christi doctrina conamur adglutinare): tanto varrebbe mescolare l'acqua col fuoco". ${ }^{47}$

Così la società cristiana - osserva Erasmo con accenti cupi - è l'esatto contrario di quella città celeste, fondata sulla "summa concordia", che Cristo aveva posto come modello per la sua Chiesa. E qui Erasmo, per rendere più efficace la sua denuncia, inventa un breve racconto, il cui protagonista è un viaggiatore "proveniente da quelle città della luna immaginate da Empedocle, o da uno degli innumerevoli mondi escogitati da Democrito": un extra-terrestre, insomma, antenato del voltairiano Micromégas... Informato dell'esistenza sulla terra di una creatura più elevata di tutte le altre, perché in possesso di un'anima fatta ad immagine di Dio, l'ospite spaziale si ragguaglia sugli insegnamenti che Cristo ha dato a tale creatura e quindi si sceglie un buon osservatorio, onde "verificare con gli occhi le informazioni acquisite per via orale". Da lì vedrà che fra tutti gli animali, che seguono generalmente le leggi di natura, ve n'è uno solo che "ingaggia con i suoi simili traffici e mercati, zuffe e guerre (unum animal inter se cauponari, negociari, rixari, belligerari)". Osservando poi da vicino la tanto celebrata comunità dei cristiani, che stando all'insegnamento di Cristo dovrebbe essere un "angelicae civitatis simulacrum", l'esterrefatto viaggiatore "Finirà, direi, per localizzare la società cristiana in una qualsiasi parte del mondo, fuorché in quella (an non ubivis potius iudicabit habitare christianos quam in hisce regionibus), che è teatro di tanta sfacciata opulenza, dissipazione, libidine, superbia, dispotismo, ambizione, frode, invidia, ira, discordie, risse, battaglie, guerre, sconvolgimenti - insomma una cloaca di tutti i vizi che Cristo ripudia, cose da Turchi o Saracini, e forse peggio (breviter omnium rerum quas Christus damnat maiorem pene Lernam quam apud illos Turcas aut Saracenos?)". ${ }^{48}$

\footnotetext{
47 Adagia, p. 240-241. ma v. pure p. 80-81 (Sileni Alcibiadis), ove si ricorda che "Aristotele cataloga fra i beni, che non toccano strettamente l'uomo, la ricchezza" (cfr. Polit., 1323a), ponendola per in fondo alla graduatoria, mentre il "vulgus" valuta massimamente proprio chi detiene ricchezze.

48 Adagia, p. 238-241.
} 
È l'immagine speculare (e rovesciata) dell'isola di Utopia, ove invece l'"angelicae civitatis simulacrum" è stato realizzato da una popolazione che, al pari dei Turchi e dei Saraceni, non appartiene alla Chiesa di Cristo... Ed è soprattutto in quell'avverbio ubivis che si pu cogliere l'idea - una vera intuizione o illuminazione - cui Moro forse s'ispirò per il suo "libellus vere aureus, nec minus salutaris quam festivus": la descrizione del Luogo-che-non-c'è, compiuta da un altro viaggiatore (questa volta un marinaio e non uno spaziale) che racconta cose dell'altro mondo, suona come una risposta filosofico-ironica. al desolato appello ad un Luogo-ovunqueesso-sia... I 'prestito' di cui Moro potrebbe essere debitore all'amico Erasmo appare qui evidente, anche se è difficile ricostruirne l'esatto ammontare; ma è un prestito che, come nella parabola dei talenti, il "morosophus" Tommaso Moro seppe mettere ben a frutto. 